\title{
Delayed LMS Algorithm for Ballistocardiogram Biomedical Signal
}

\author{
Manjula B M, Prasantha H S, Goutham M A
}

\begin{abstract}
The Ballisto-cardiogram (BCG) is a biomedical signal which is basically a measure of ballistic forces on heart. Just like ECG to detect the abnormalities in heart Ballisto-cardiography technique is used extensively now a day in research to analyze the abnormalities of the patient. When the blood pumps from heart to different parts of the body is represented in the form of graph for each heart beat. The frequency of Ballisto-cardiography signal is

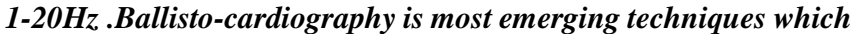
is used to test the diseases related to heart called as cardiovascular disease. Various devices [1] like chairs, beds and weighing scales are projected to improvise the extraction of the BCG, but noise is one of the main issues with this BCG signal processing, noise is generated because of motion artifacts, shaking of the devices or may be power line noise. This noise [2] affects the quality of signal which we need to test. In order to overcome such issue this paper proposes a new architecture making use of LMS filtering algorithm Here weight update algorithm is used to update the error in extracted signal. The architecture proposed here includes FIR filter and also error computation blocks. Here the author has implemented 5-tap filtering algorithm. MATLAB and system generators are used to carry out the work.
\end{abstract}

Keywords: Ballistocardiogram signal, Least Mean Square, FIR filter,weight update

\section{INTRODUCTION}

One of the greatest challenge in the society is cardiovascular disease. The effect of heart disease [3] and its causes are increasing rapidly. As per A.H.A by 2025, 42.5 percentage of people of United States will suffer with heart disease and the cost is increases rapidly and it will reaches to $\$ 900$ billion [4]. In order to manage cardiovascular disease there is an alternate method just like ECG to find the abnormalities in heart that is ballisto-Cardiography approach. To get accuracy in measurement, variety of techniques are proposed those are using beds, chairs and weighing scales all these methods gives results as output of the cardiac. [5] Motion artifacts which is caused due to vibrations are one of the main issues in BCG signal processing. Motion artifacts include eye blinking, head movement, muscle contraction which affects

Revised Manuscript Received on July 10, 2020.

* Correspondence Author

Manjula B M*, Department of ECE, Nitte Meenakshi Institute of Technology, Bangalore, India. E-mail: manjula.nmit@gmail.com

Prasantha H S, Department of ECE, Nitte Meenakshi Institute of Technology, Bangalore, India.

Goutham M A, Department of ECE, Adi Chunchanagiri Institute of Technology, Chikkamagalur, India.

(C) The Authors. Published by Blue Eyes Intelligence Engineering and Sciences Publication (BEIESP). This is an open access article under the CC BY-NC-ND license (http://creativecommons.org/licenses/by-nc-nd/4.0/) the quality of the signal in diagnosis. Hence it is necessary to eliminate such artifacts and noise. Hence this paper proposes an adaptive filter using LMS algorithm to eliminate the noise present in the data. The algorithm concentrates on echo in the signal, noise in signal, identification of channel and channel equalization etc. FIR is well known filtering technique for signal filtering. B. Widrow et al. [10] proposed an adaptive least mean square algorithm using weight up-date method. LMS adaptive technique is less complex l implement. It gives better performance for the convergence issues [11]. Delayed LMS adaptive Filter (DLMS) is another approach, to find the improved performance of filtering process. Critical path delay is one of the disadvantages of this approach and it can be overcome by designing pipelined architecture. [12].

\section{RELATED WORK}

In this work [1] the author gives an efficient algorithm to filter noise in the BCG signal. Adaptive weight scheme for 2Tap signal filtering includes FIR filtering and error computation blocks are used here to improve efficiency of weight update process. Work presented here is implemented using MATLAB tool. The results are examined using the dataset of heart rate. The obtained results show accuracy in the result by reducing noise in the signal. In work [2] Noise present in the signal can be eliminated by Adaptive Noise Cancellation technique. The simulation result obtained shows the merits of adaptive noise cancellation. As per the result obtained the noise was suppressed but there will be a little signal distortion. In Paper [3] the author presented an efficient architecture for getting lower adaptation-delay using adaptive filter. Partial product generator method is used in this paper to get the result. The author proposed an optimized pipelining technique across the time--consuming combinational blocks of the structure The results shows that the proposed design gives around $14 \%$ less energy.delay-product (EDP) and around 17\% less area, delay.product (ADP) than the method existed.The author proposed an efficient fixed-point algorithm to implement the architecture, and obtained an expression for steady-state error. The Paper shows steady-state and mean squared error obtained from analytical result that is matches with simulation result obtained. In [4] an enhanced building design to execute deferred slightest mean square versatile channel is discussed. The Least Mean Square algorithm is most prominent and widely used technique for filtering. As per author the Least Mean Square configuration offers less delay and less energy delay.

Blue Eyes Intelligence Engineering and Sciences Publication (C) Copyright: All rights reserved.

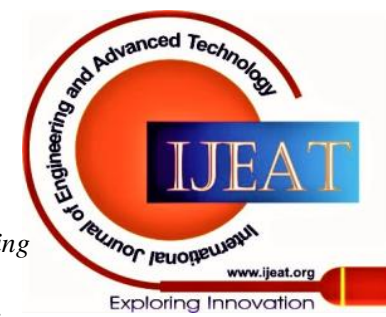




\section{Delayed LMS Algorithm for Ballistocardiogram Biomedical Signal}

In proposed versatile technique the configuration is stretched out by supplanting LMS calculation to Recursive slightest squares calculation which is an improved execution. The implemented design incorporated fundamentally less adjustment postpones and gave critical sparing of ADP and EDP.

\section{IMPLEMENTATION OF DELAYED LMS ALGORITHM}

As per the literature we have implemented the different types of adaptive filter [8] and the results are compared with each other and found that LMS adaptive filter is the most suitable one to get better noise reduction. But the disadvantage of LMS adaptive filter has higher delay. But this delay can be reduced by using pipelined architecture. The LMS algorithm is widely used technique of adaptive filtering. Here each data signal time interval is calculated and eliminated from desired signal. The error signal is required to updates the coefficients of the filter before arrival of the next sample [11]

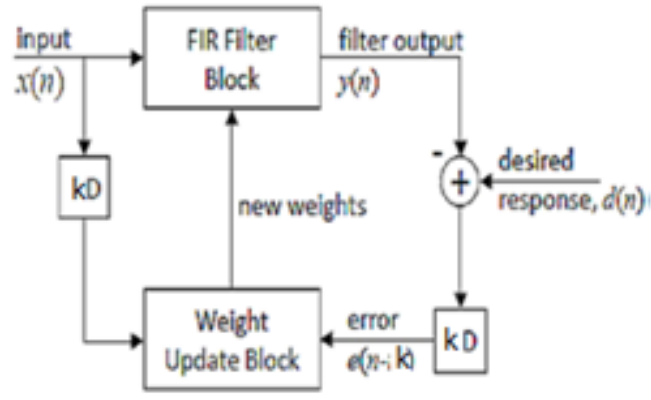

Figure 1:Conventional Adaptive filter

In DLMS algorithm, the error corresponding to the (n-m) ${ }^{\text {th }}$ iteration , $\mathrm{e}(\mathrm{n}-\mathrm{m})$ is the most recent error corresponding to $\mathrm{n}^{\text {th }}$ iteration, e(n) is required to update the current weights. For DLMS filter, weight update equation are

$$
\begin{aligned}
& \mathrm{y}(\mathrm{k})=\mathrm{w}_{\mathrm{n}}^{\mathrm{T}} \cdot \mathrm{x}(\mathrm{k}) \\
& \mathrm{e}(\mathrm{k})=\mathrm{d}(\mathrm{k})-\mathrm{y}(\mathrm{k})(2) \\
& \mathrm{w}_{\mathrm{k}+1}=\mathrm{w}_{\mathrm{k}} \cdot \beta \cdot \mathrm{x}(\mathrm{k}) \cdot \mathrm{e}(\mathrm{k}) \ldots
\end{aligned}
$$

Where

$$
\begin{aligned}
& X(k)=[x(k), x(k-1), x(k-2) \ldots x(k-N+1)]^{T} \\
& W_{k}=\left[w_{k}(0), w_{k}(1), w_{k}(2) \ldots \ldots \ldots w_{k}(N-1)\right]^{T}
\end{aligned}
$$

Here

$\mathrm{X}(\mathrm{k})$ : is input signal vector

$\mathrm{W}_{\mathrm{k}}$ : Weight vector of the $\mathrm{N}^{\text {th }}$ order LMS filter

$\mathrm{d}(\mathrm{k})$ : Desired signal

$\mathrm{y}(\mathrm{k})$ : Output of the filter

$\mathrm{N}$ : Order of LMS filter

$\beta$ :Step size or convergence-factor.

$\mathrm{e}(\mathrm{k})$ : Error to update the weights for use in the next iteration.

\section{PROPOSED WORK}

In traditional delayed LMS filter, adaptation delay results in delay which is introduced by complete filter structure, which includes weight adaptation and FIR filtering. Instead of using single delay, we can decompose the adaptation delay into two parts, namely (i) delay required for FIR filtering (ii) delay required to update the weight, which is illustrated in figure 2 LMS algorithm is designed using delayed LMS filter. The error computation block contains both computation block and final subtraction block that computes the feedback error. The error computation path latency is reduced by employing this structure. For delay of $n 1$ cycles, the error at the $n^{\text {th }}$ cycle is $\mathrm{e}(\mathrm{n}-\mathrm{k} 1)$, and it is used along with input samples and the delay required is $\mathrm{k} 1$ cycles to generate required weight increment term. The mathematical equation for weight update of proposed LMS is

$\mathrm{w}_{\mathrm{k}+1}=\mathrm{w}_{\mathrm{k}}+\mu \mathrm{e}\left(\mathrm{k}-\mathrm{k}_{1}\right) \cdot \mathrm{x}\left(\mathrm{k}-\mathrm{k}_{1}\right)$

$\mathrm{e}\left(\mathrm{k}-\mathrm{k}_{1}\right)=\mathrm{d}\left(\mathrm{k}-\mathrm{k}_{1}\right)-\mathrm{y}\left(\left(\mathrm{k}-\mathrm{k}_{1}\right)\right.$

$\mathrm{y}(\mathrm{k})=\mathrm{w}_{\mathrm{k}-\mathrm{k} 2}^{\mathrm{T}} \cdot \mathrm{x}(\mathrm{k})$

$\mathrm{y}(\mathrm{k})$ : Output of the filter

$\mathrm{w}_{\mathrm{k}}$ : Present weight

$\mathrm{w}_{\mathrm{k}+1}$ : Updated weight of adaptive filter

Here, $\mathrm{k} 1$ times delayed error is utilized for weight adaptation and k2 times delayed weights are used by the filtering unit. This method reduces the adaptation by $\mathrm{k} 2$ cycles.

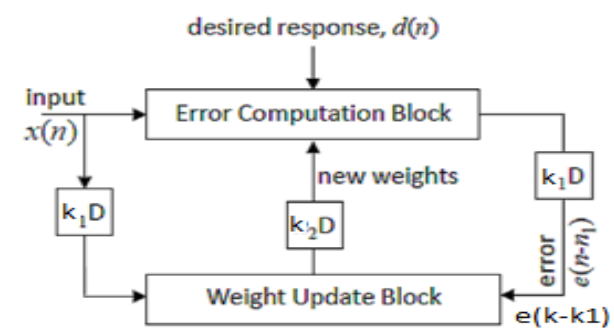

Figure 2: Proposed delayed LMS adaptive filter.

The flow chart of the proposed filter is shown in figure 3.

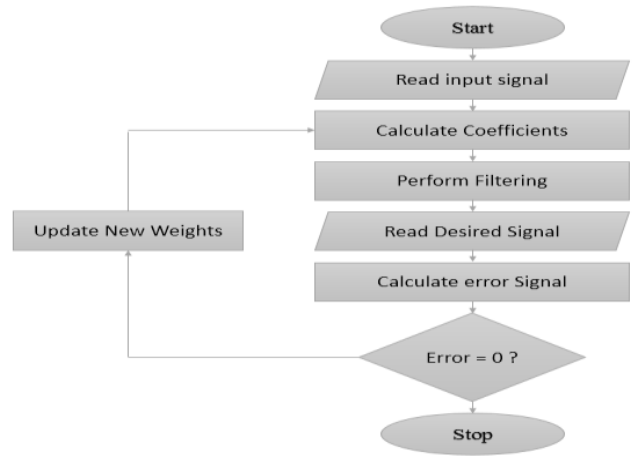

Figure 3: Flow chart of the proposed Filter

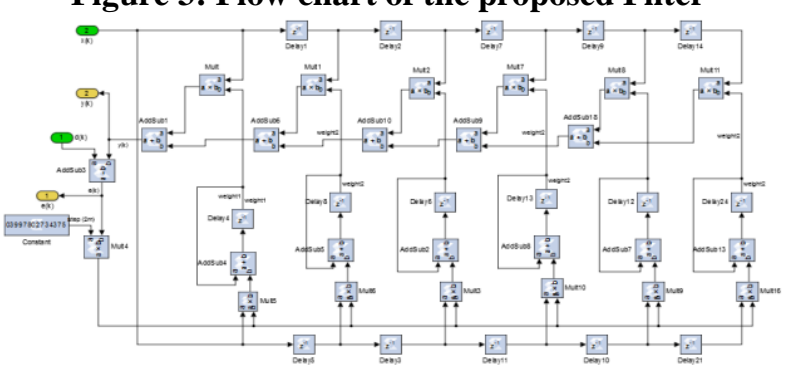

Figure 4: System Generator implementation of LMS Adaptive filter of order 5

Published By:

Blue Eyes Intelligence Engineering and Sciences Publication 
The designed delayed LMS algorithm is implemented in system generator platform and is shown in figure 5 . The filter block is implemented using few sets of multiplier, adders and delay elements. The hardware co-simulation is performed using Virtex II Pro 2vp30ff896-7 FPGA and is shown in figure 5 and the corresponding waveforms is shown in figure6. The system is designed and implemented for filter order of 2 and 5.

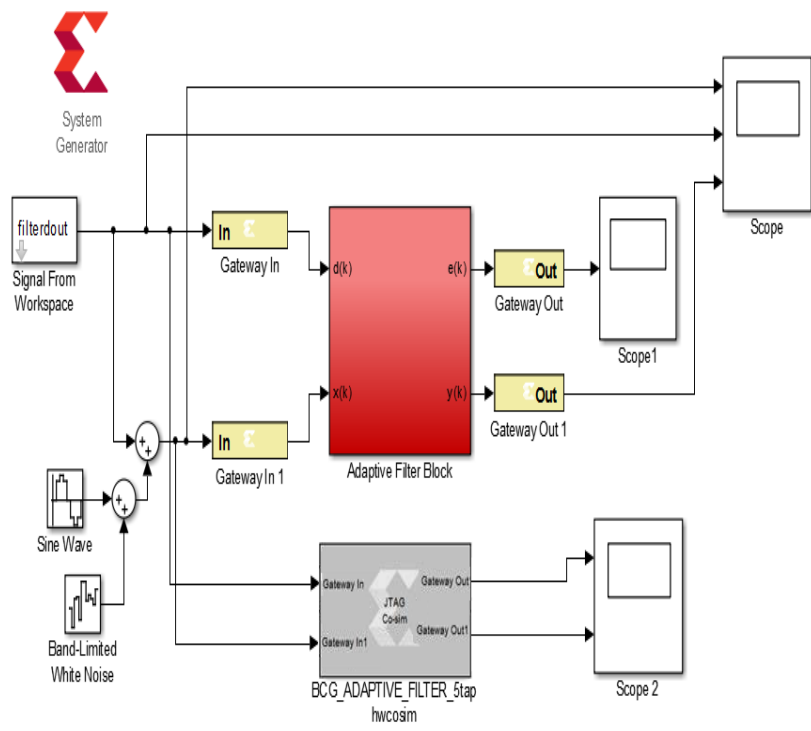

Figure 5: Hardware Co-simulation of LMS Filters

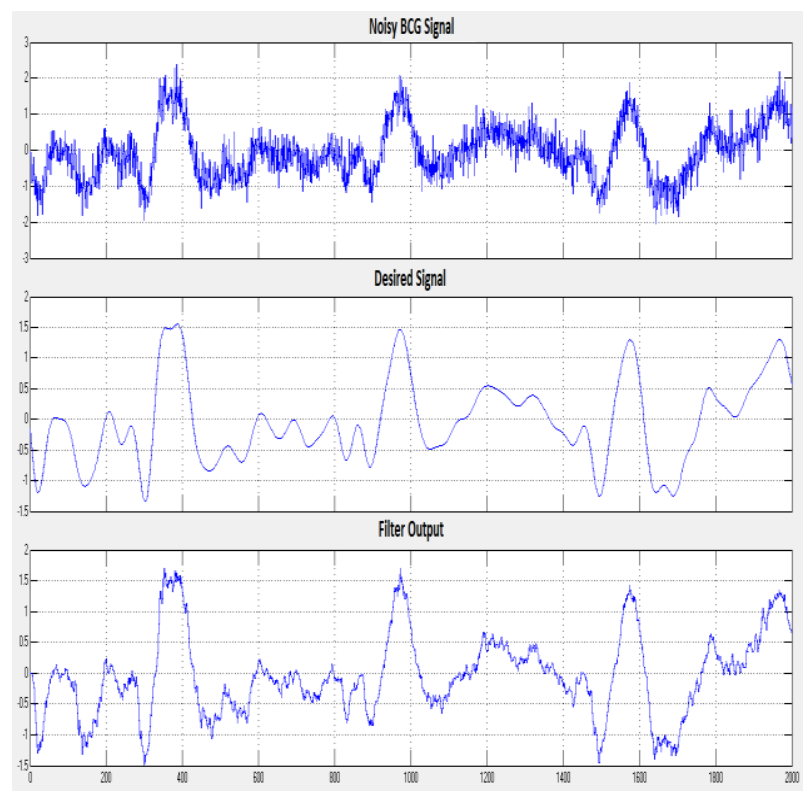

Figure 6: Results of LMS filter of order 5

The output obtained from the filter is pure BCG and it has suppressed all the interferences and noise in it. The RTL schematic of the designed system is shown in figure 7 . Extracted device utilization summery is shown in table 1.

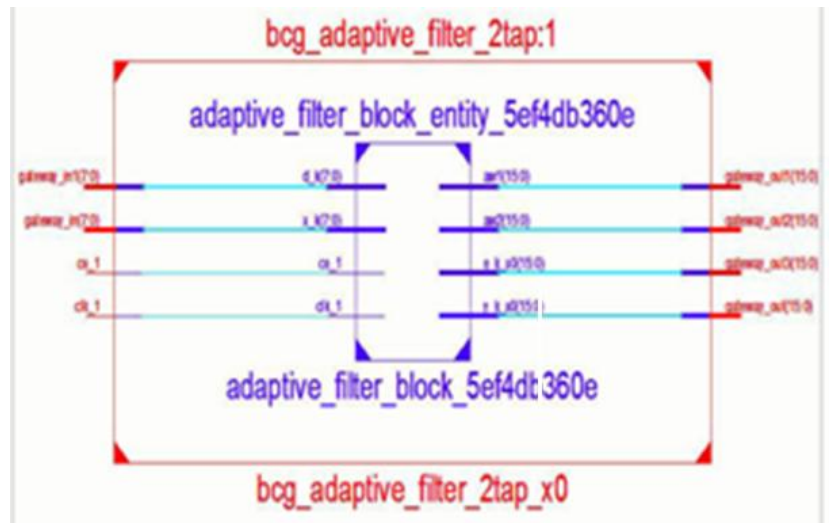

Figure 7:RTL Schematic of DLMS adaptive filter

Table1 :Device Utilization Summary

\begin{tabular}{|c|c|c|c|}
\hline $\begin{array}{c}\text { Logic } \\
\text { Utilization }\end{array}$ & Used & Available & Utilization \\
\hline No.of Slices & 106 & 35840 & $0 \%$ \\
\hline $\begin{array}{c}\text { No.of Slice } \\
\text { FF }\end{array}$ & 67 & 71680 & $0 \%$ \\
\hline $\begin{array}{c}\text { No.of } 4 \text { i/p } \\
\text { LUT }\end{array}$ & 185 & 71680 & $0 \%$ \\
\hline $\begin{array}{c}\text { No of } \\
\text { bounded } \\
\text { IOBs }\end{array}$ & 81 & 768 & $10 \%$ \\
\hline No of GCLKs & 01 & 32 & $3 \%$ \\
\hline No.of DSPs & 07 & 80 & $8 \%$ \\
\hline
\end{tabular}

\section{SIMULTION RESULT AND ANALYSIS}

Simulation parameters considered for BCG signal filtering are given in table 2 .

Table II: Simulation Parameters

\begin{tabular}{|c|c|}
\hline Parameters Considered & Values \\
\hline Total beats considered & 05 \\
\hline threshold of peak detection & 0.6 \\
\hline Left side window & 0 \\
\hline Right side window & 700 \\
\hline Sampling frequency & 1000 \\
\hline Stop band frequency & 0.22 \\
\hline Passband Frequency & 01 \\
\hline
\end{tabular}

Simulation parameters for 2-tap and for 5 tap filtering operation.

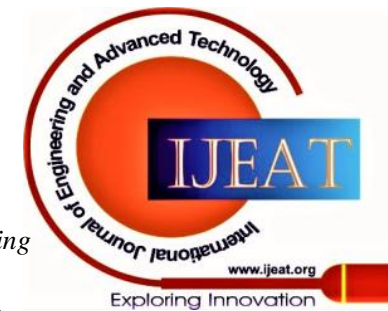




\section{Delayed LMS Algorithm for Ballistocardiogram Biomedical Signal}

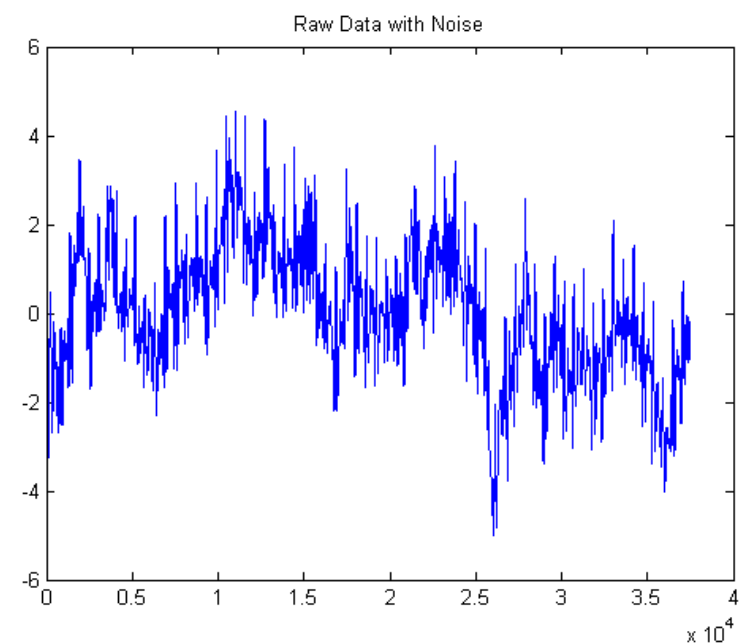

Figure 8: Observed data

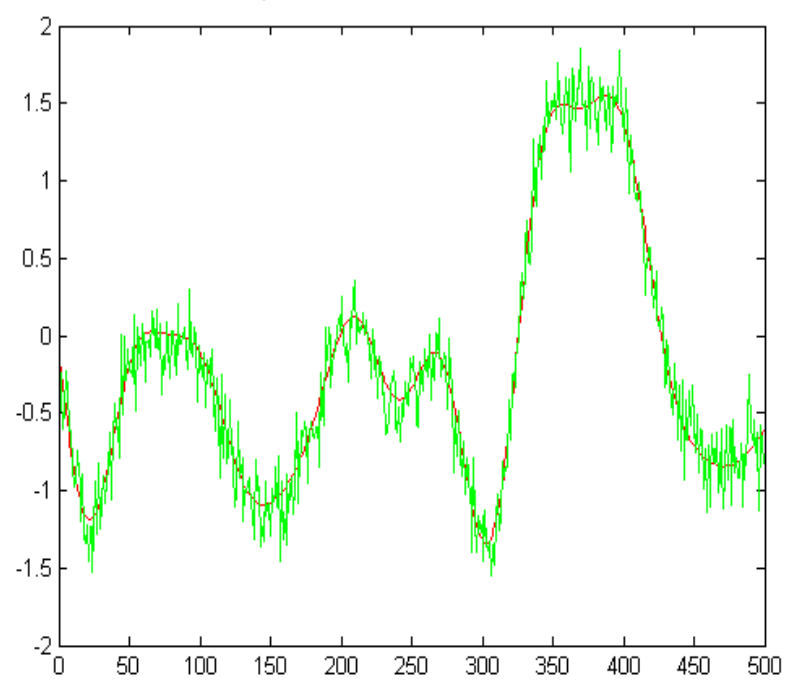

Figure.9: Noisy Data

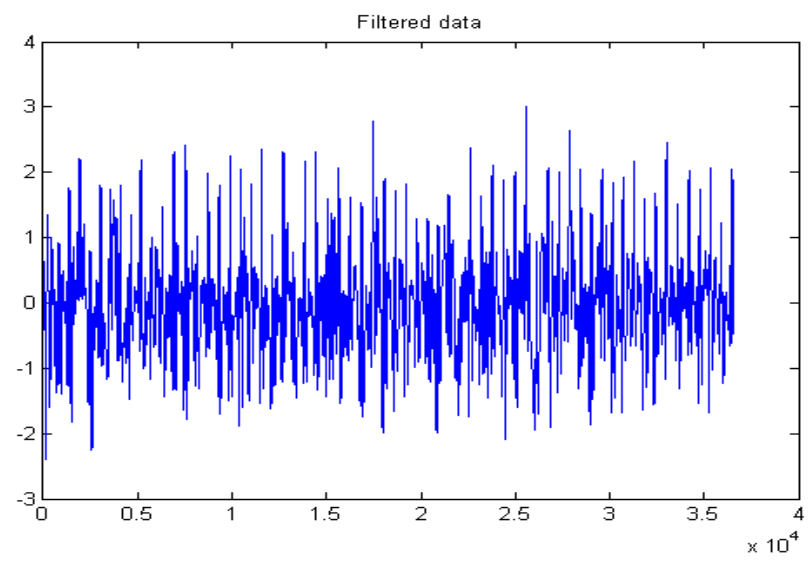

Figure.10. Filtered data

Noise is mainly because of vibrations of the device. The noisy data is processed in to MATLAB to get better filtered output. The result of this is shown in figure 10 .

\section{CONCLUSION}

The paper deals with an efficient algorithm for BCG signal filtering. BCG filtering algorithm is implemented using an adaptive weight update scheme for 2 Tap \& 5Tap. The algorithm uses FIR filtering and also error computation blocks to increase the efficiency of the said procedure. The work is carried out using MATLAB software tool and tested using heart rate with filtering parameters. The results obtained shows the efficiency in terms of reduction of error and artifacts .

\section{REFERENCES}

1. O. T. Inan, M. Etemadi, R. M. Wiard, L. Giovangrandi, and G. T. A. Kovacs, "Robust ballistocardiogram acquisition for home monitoring," Physiol. Meas., vol. 30, p. 169, 2009.

2. Shubhra Dixit, Deepak Nagaria "LMS Adaptive Filters for Noise Cancellation: A Review" Vol. 7, No. 5, October 2017, pp. 2520 2529 International Journal of Electrical and Computer Engineering (IJECE)

3. Area-Delay-Power Efficient Fixed-Point LMS Adaptive Filter with Low Adaptation-Delay Pramod Kumar Meher, Senior Member, IEEE and Sang Yoon Park, Member, IEEE transactions on very large scale integration (vlsi) systems.

4. Mr. M Basha ,Mr. V Santhosh Kumar" Adaptive Filter with Low Adaptation Delay Improving the LMS Fixed Point" Internationa Journal of Ethics in Engineering \& Management Education, Volume 3, Issue 6, June 2016)

5. P. K. Meher and Sang Yoon Park, "Area-Delay-Power Efficient Fixed-Point LMS Adaptive Filter With Low Adaptation-Delay," in IEEE Transactions on Very Large Scale Integration (VLSI) Systems, vol. 22, no. 2, pp. 362-371, Feb. 2014

6. Santosh Bhalke,Manjula B.M,Chirag Sharma,"FPGA Implementation of Efficient FIR filter With Quantised fixed point coefficients,2013 international conference on emerging trends in communication, control, signal processing and computing applications (c2spca)

7. Ms.Manjula B.M , Dr.Chirag Sharma ," FPGA Implementation of BCG Signal Filtering Scheme by using Weight Update Process" Indonesian Journal of Electrical Engineering and Computer Science Vol. 4, No. 2, November 2016, pp. $373 \sim 382$ DOI: 10.11591/ijeecs.v4.i2.pp373-382

8. Nitesh , Manjula B.M.” Data Processing of Ballistocardiogram Signal using Adaptive Filter" international journal of innovative research in electrical, electronics, instrumentation and control engineering vol. 4 , issue 5, may 2016, doi 10.17148/ijireeice.2016.4563

9. Manjula B M ,Chirag Sharma," Ballistocardiography signal filtering using adaptive least mean square algorithm"IEEE international conference on recent trends in electronics information communication technology, may 20-21, 2016, india, doi: 10.1109/rteict.2016.7807797

10. Widrow and S. D. Stearns, Adaptive signal processing. Englewood Cliffs, NJ: Prentice-Hall, 1985.

11. P. K. Meher and Sang Yoon Park, "Area-Delay-Power Efficient Fixed-Point LMS Adaptive Filter With Low Adaptation-Delay," in IEEE Transactions on Very Large Scale Integration (VLSI) Systems, vol. 22, no. 2, pp. 362-371, Feb. 2014

12. P. K. Meher and S. Y. Park, "Low adaptation-delay LMS adaptive filter part-I: Introducing a novel multiplication cell," in Proc. IEEE International Midwest Symposium on Circuits and Systems (MWSCAS), Aug. 2011.

\section{AUTHORS PROFILE}

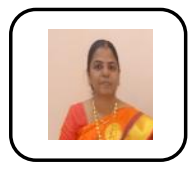

Dr. Manjula B M received Bachelor degree from Mangalore University and Master Degree from V.T.U, Belgaum, and completed Ph.D from University of Mysore. She has 19+ years of teaching experience. She is currently working as a Associate Professor in the department of Electronics and Communication Engineering, Nitte Meenakshi Institute of Technology,

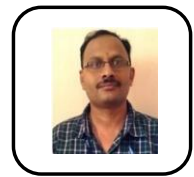

Dr. Prasantha H S received Bachelor degree from Bangalore University, Master Degree from V.T.U, Belgaum, and Ph.D from Anna University, Chennai, in the area of Multimedia and Image Processing. He has 19+ years of teaching and research experience. His research interest includes Multimedia and Signal Processing. He is currently guiding students for their research program under VTU. Currently, he is working as a Professor in the department of Electronics and Communication Engineering, Nitte Meenakshi Institute of Technology, Bangalore working as Assistant Professor in Department of of Electronics and Communication Engineering, Nitte Meenakshi Institute of Technology, Bangalore.

Published By:

Blue Eyes Intelligence Engineering

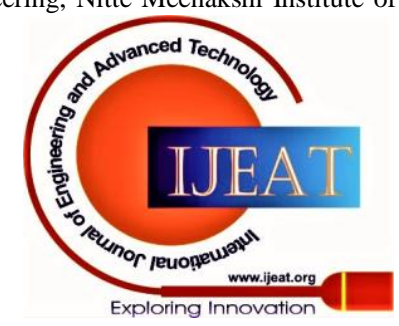


Dr. Goutham M.A received Ph.D from Jadhavpur University, Kolkotha, in the area of Image Processing. He has 20+ years of teaching and research experience. He is currently guiding students for their research program under VTU and Mysore university.Currently, he is working as a Professor and Head in the department of Electronics and Communication Engineering, Adichunchanagiri Institute of Technology, Chikkamangalore. 